\title{
Evidence for Topological Edge States in a Large Energy Gap near the Step Edges on the Surface of $\mathrm{ZrTe}_{5}$
}

\author{
R. Wu, ${ }^{1,2}$ J.-Z. Ma, ${ }^{1}$ S.-M. Nie, ${ }^{1}$ L.-X. Zhao, ${ }^{1}$ X. Huang, ${ }^{1,2}$ J.-X. Yin, ${ }^{1,2}$ B.-B. Fu, ${ }^{1}$ P. Richard, ${ }^{1,3}$ G.-F. Chen, ${ }^{1,3}$ \\ Z. Fang, ${ }^{1,3}$ X. Dai, ${ }^{1,3}$ H.-M. Weng, ${ }^{1,3,}{ }^{*}$ T. Qian, ${ }^{1, \dagger}$ H. Ding, ${ }^{1,3}$ and S. H. Pan ${ }^{1,2,3, \$}$ \\ ${ }^{1}$ Beijing National Laboratory for Condensed Matter Physics and Institute of Physics, \\ Chinese Academy of Sciences, Beijing 100190, China \\ ${ }^{2}$ Department of Physics and Texas Center for Superconductivity, University of Houston, \\ Houston, Texas 77204, USA \\ ${ }^{3}$ Collaborative Innovation Center of Quantum Matter, Beijing, China
}

(Received 31 December 2015; published 10 May 2016)

\begin{abstract}
Two-dimensional topological insulators with a large bulk band gap are promising for experimental studies of quantum spin Hall effect and for spintronic device applications. Despite considerable theoretical efforts in predicting large-gap two-dimensional topological insulator candidates, none of them have been experimentally demonstrated to have a full gap, which is crucial for quantum spin Hall effect. Here, by combining scanning tunneling microscopy/spectroscopy and angle-resolved photoemission spectroscopy, we reveal that $\mathrm{ZrTe}_{5}$ crystal hosts a large full gap of $\sim 100 \mathrm{meV}$ on the surface and a nearly constant density of states within the entire gap at the monolayer step edge. These features are well reproduced by our first-principles calculations, which point to the topologically nontrivial nature of the edge states.

DOI: 10.1103/PhysRevX.6.021017

Subject Areas: Condensed Matter Physics,

Topological Insulators
\end{abstract}

\section{INTRODUCTION}

A topological insulator (TI) is a novel state of matter characterized by an energy gap in the bulk and gapless Dirac fermionic states connecting the valence and conduction bands on the boundary. For two-dimensional (2D) TIs, time-reversal symmetry requires that electrons propagating in opposite directions have opposite spins in the onedimensional (1D) edge state. Backscattering is prohibited as long as time-reversal symmetry is not broken, leading to dissipationless transport edge channels and quantum spin Hall (QSH) effect [1,2]. These exotic properties suggest tantalizing possibilities in electronic device applications. Although considerable theoretical efforts have predicted many 2D TI candidates in recent years [3-17], only a few systems, such as the quantum wells of $\mathrm{HgTe} / \mathrm{CdTe}$ and InAs/GaSb, have been demonstrated experimentally to display the QSH effect $[18,19]$. However, in both of these heterojunction materials the QSH effect shows up only at ultralow temperatures due to a small energy gap on the order

\footnotetext{
* Corresponding author. hmweng@iphy.ac.cn

Corresponding author. tqian@iphy.ac.cn

Corresponding author. span@iphy.ac.cn

Published by the American Physical Society under the terms of the Creative Commons Attribution 3.0 License. Further distribution of this work must maintain attribution to the author(s) and the published article's title, journal citation, and DOI.
}

of meV. Highly precise material control is also required to fabricate such quantum well structures. These extreme requirements have obstructed their further studies and possible applications. Thus, seeking for desirable 2D TIs with a large gap is becoming urgently important. The $\mathrm{Bi}$ (111) bilayer films have been demonstrated to host topological edge states within a large gap [20,21]. However, strong coupling to the states of the substrates leads to a finite density of states (DOS) within the gap in the area away from the edges, which makes measurements of the QSH effect and device applications nearly infeasible.

An alternative way to achieve 2D TIs with topological edge states is to utilize the step edges on the surface of a cleaved single crystal. Such topological edge states have been observed on the surfaces of $\mathrm{Bi}$ and $\mathrm{Bi}_{14} \mathrm{Rh}_{3} \mathrm{I}_{9}$ crystals $[22,23]$, but still with an undesired finite DOS inside the energy gap in the area away from the step edges. In principle, the surface with a step edge can be viewed as a monolayer being placed on top of a "substrate." For some layered materials with very weak interlayer coupling, it is possible for the step edge on a clean surface to have similar topological edge states, provided the monolayer system is a good 2D TI. In order for the topological edge states to survive at the step edge, another important criteria must be satisfied besides the weak enough interlayer coupling: the bulk material must be a weak TI, so that there will be no topological surface states on the surface perpendicular to the stacking direction. Otherwise, if the three-dimensional (3D) material is a strong TI, the original edge states at the step edge will hybridize with the topological surface states and lose their 1D nature completely. 
In this paper, we show that a $\mathrm{ZrTe}_{5}$ single crystal satisfies all the aforementioned necessary conditions, and therefore is able to accommodate the topological edge states at the step edge on the surface. First of all, $\mathrm{ZrTe}_{5}$ is a layered material and its monolayer is predicted to be a good 2D TI with an energy gap of $100 \mathrm{meV}$ [24]. Secondly, the interlayer coupling of the $\mathrm{ZrTe}_{5}$ crystal is van der Waals type and is very weak compared to the intralayer bonding strength. Thirdly, $\mathrm{ZrTe}_{5}$ crystal is a weak TI with no topological surface states on the terminating surface.

By using scanning tunneling microscopy/scanning tunneling spectroscopy (STM/STS) and angle-resolved photoemission spectroscopy (ARPES), we systematically investigate the electronic states on the surface and at a monolayer step edge of $\mathrm{ZrTe}_{5}$ crystals. We observe a large energy gap of $\sim 100 \mathrm{meV}$ between the nearly lineardispersive "conelike" valence and conduction bands at the Brillouin zone center. The slight $k_{z}$ dispersion indicates that the interlayer coupling is very weak. The high-resolution STS spectrum shows no DOS inside the entire energy gap on the surface area far away from the step edge, indicating that the bulk material of $\mathrm{ZrTe}_{5}$ is in a weak TI phase. More significantly, we observe at the edge of a monolayer terrace a finite DOS, which is nearly constant inside the energy gap. Such edge states are well reproduced by our single-layer ribbon calculations that show topologically nontrivial Diraclike edge states exist inside the large energy gap.

\section{EXPERIMENTAL AND COMPUTATIONAL METHODS}

Single crystals of $\mathrm{ZrTe}_{5}$ are grown by the chemical vapor transport method. Stoichiometric amounts of $\mathrm{Zr}$ (powder 99.2\%, Hf nominal 4.5\%) and Te (powder 99.999\%) are sealed in an evacuated quartz ampoule with iodine $(7 \mathrm{mg} / \mathrm{mL})$ and placed in a two-zone furnace. A typical temperature gradient from $480{ }^{\circ} \mathrm{C}$ to $400^{\circ} \mathrm{C}$ is applied. After one month, long ribbon-shaped single crystals are obtained. STM measurements are carried out on $\mathrm{ZrTe}_{5}$ single crystal samples that are cleaved in situ at $\sim 20 \mathrm{~K}$, and all measurements are performed at $4.3 \mathrm{~K}$. ARPES measurements are performed at the "Dreamline" beam line of the Shanghai Synchrotron Radiation Facility (SSRF) with a Scienta D80 analyzer. The energy and angular resolutions are set to $15-30 \mathrm{meV}$ and $0.2^{\circ}$, respectively. The samples for ARPES measurements are cleaved in situ and measured in a temperature range between $24 \mathrm{~K}$ and $200 \mathrm{~K}$ in a vacuum better than $5 \times 10^{-11}$ Torr.

The Vienna ab initio simulation package (VASP) $[25,26]$ is employed for first-principles calculations. The generalized gradient approximation of Perdew-Burke-Ernzerhof type [27] is used for the exchange-correlation potential. Spinorbit coupling is taken into account self-consistently. The cutoff energy for plane wave expansion is $500 \mathrm{eV}$ and the $k$-point sampling grids for different structures are tested to be dense enough. The atomic structure and the lattice constants $a=3.987 \AA, b=14.502 \AA$, and $c=13.727 \AA$, determined by powder diffraction [28], are used in our calculations. The $\mathrm{ZrTe}_{5}$ layers are stacked along the $b$ axis. Band topology analysis indicates that increasing the interlayer distance by only $0.25 \AA(\sim 3.4 \%)$ will tune the material from strong TI to weak TI [24]. Such a small change is within the error in determining the lattice constants by generalized gradient approximation calculations. In this work, we take a slightly increased interlayer distance to mimic the weak TI phase, which is consistent with our experimental observations. A vacuum $15 \AA$ thick is used to minimize the interactions between the layers or ribbons and its periodic images. To largely reproduce the realistic (010) surface of the sample, we build a slab model with 9 unit cell thick along the $b$ axis $[29,30]$. To study the edge states along the $a$ axis, a single ribbon with 9 unit cell wide along the $c$ axis, terminated by a $\mathrm{Te}_{2}$ zigzag chain and a $\mathrm{ZrTe}_{3}$ chain on each side, is calculated.

\section{RESULTS}

$\mathrm{ZrTe}_{5}$ crystals have an orthorhombic layered structure characterized by space group $\mathrm{Cmcm}$. As shown in Figs. 1(a) and 1(b), the triple prisms of $\mathrm{ZrTe}_{3}$ form chains running along the $a$ axis, and the adjacent chains are linked via parallel zigzag chains of Te atoms to form a 2D sheet of $\mathrm{ZrTe}_{5}$ in the $a-c$ plane with the lattice constants $a=3.987 \AA$ and $c=13.727 \AA$. The sheets of $\mathrm{ZrTe}_{5}$ stack along the $b$ axis with a spacing of $7.251 \AA$, forming a layered structure. The adjacent $\mathrm{ZrTe}_{5}$ layers are coupled via weak van der Waals forces, so that the cleavage mostly takes place between them.

Figure 1(c) displays a STM constant-current topographic image of an exposed $\mathrm{ZrTe}_{5} a-c$ (010) surface after cleavage. We observe bright stripes parallel to each other with a separation of $13.9 \AA$, which matches the lattice constant $c$. The inset at the top right-hand corner of Fig. 1(c) indicates that the stripes are constituted of dimers. The distance between the dimers along the stripe direction is $4.1 \AA$, which matches the lattice constant $a$. The distance of the two bright spots in one dimer is $2.8 \AA$, which is consistent with that of the two Te atoms at the top of a triple prism. Thus, the bright stripes represent the $\mathrm{ZrTe}_{3}$ chains in the termination layer. No obvious reconstruction is observed on the terminating surface. The electronic structure is spatially homogeneous on a clean surface. The inset at the bottom left-hand corner of Fig. 1(c) shows the differential tunneling conductance spectrum, which is proportional to the DOS. The conductance drops to zero within an energy range of $\sim 100$ meV slightly above the Fermi energy $\left(E_{F}\right)$, demonstrating the existence of a large full energy gap on the surface of the $\mathrm{ZrTe}_{5}$ crystal.

To clarify the origin of the energy gap observed in the STS, we perform systematic ARPES measurements on the (010) surface. Figures 2(a) and 2(d) show that a holelike band along $\Gamma-X$ crosses $E_{F}$ in a "head-touching" style at low temperature, forming a small hole pocket at the Brillouin zone 
(a)

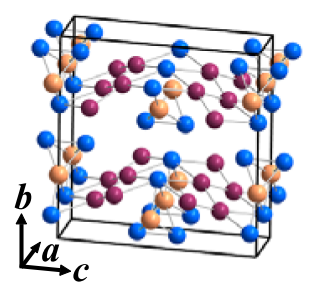

CZr $\bullet$ Te1 ๑Te2

(c)

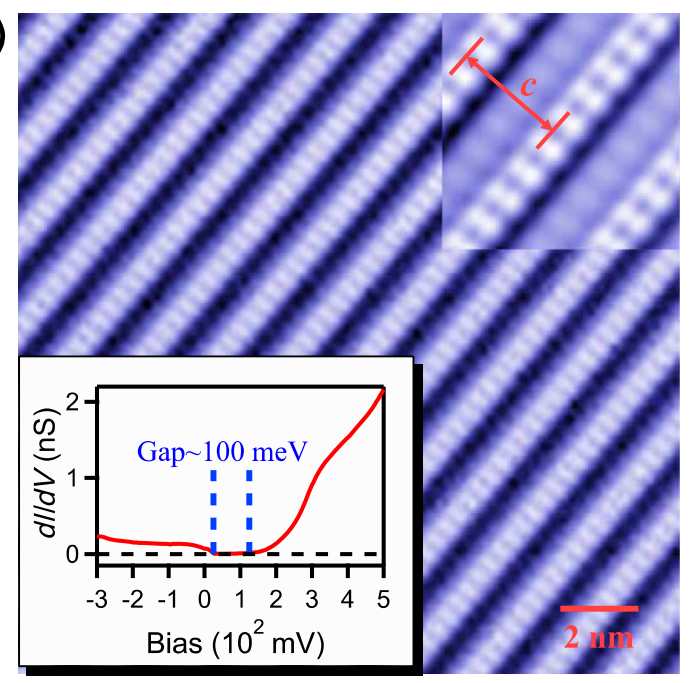

FIG. 1. (a) 3D structure of $\mathrm{ZrTe}_{5}$ crystal. (b) Top view of the single-layer structure. (c) STM constant current topographic image of the cleaved $a-c$ (010) surface of $\mathrm{ZrTe}_{5}$ crystal $(I=100 \mathrm{pA}, V=-800 \mathrm{mV})$. Right top inset: High-resolution topography $(I=50 \mathrm{pA}, V=400 \mathrm{mV})$ showing the details of the surface structure. Bottom left inset: Tunneling differential conductance spectrum on the $a-c$ surface $(I=500 \mathrm{pA}$, $V=500 \mathrm{mV}$ ) showing a large energy gap of $\sim 100 \mathrm{meV}$ above $E_{F}$ with zero conductance inside the gap.

center, as seen in the Fermi surface intensity map in Fig. 2(f). The photon energy dependence measurements displayed in Fig. 2(e) reveal that the peaks of the momentum distribution curves exhibit a slight modulation along the $k_{z}$ direction with a period of $2 \pi / b^{\prime}$, where $b^{\prime}$ is one-half of the $b$-axis lattice constant of $\mathrm{ZrTe}_{5}$. The slight $k_{z}$ dispersion indicates that the interlayer coupling is very weak.

The experimental band dispersions are consistent with the calculated valence band structure, as seen in Fig. 2(a). It is clear that $E_{F}$ lies slightly below the valence band top. This feature is consistent with the observation in the STS measurements, in which the conductance drops to zero approximately at a positive bias of $30 \mathrm{meV}$. To observe the conduction bands, we attempt to deposit potassium atoms onto the surface, which can dope electrons into the surface and thus shift the chemical potential upwards. However, the chemical potential is only shifted to slightly above the valence band top, as shown in Fig. 2(c). Further deposition has no effect on the chemical potential. Another
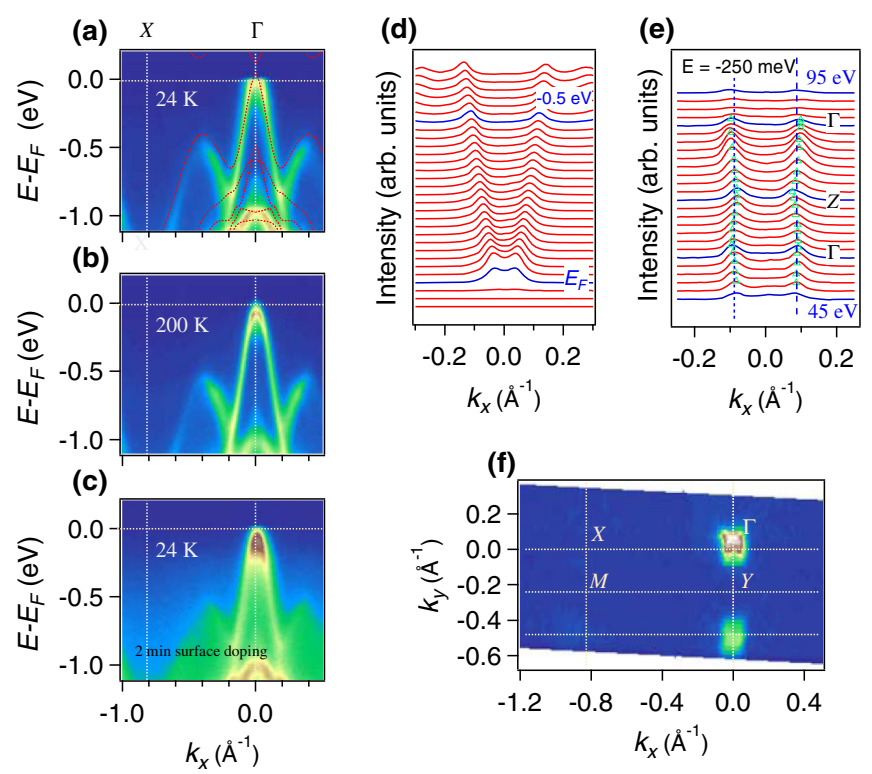

FIG. 2. Band structure of pristine $\mathrm{ZrTe}_{5}$. (a) Band dispersions along $\Gamma-X$ measured at $24 \mathrm{~K}$. For comparison, the calculated band structure along $\Gamma-X$ is plotted on top of the experimental data. The calculated bands are shifted up by $70 \mathrm{meV}$ to match the experimental data. (b) Same as (a) but recorded at 200 K. (c) Same as (a) but measured after the deposition of potassium atoms for 2 min. (d) Momentum distribution curve plot of (a) showing two peaks at $E_{F}$, confirming the $E_{F}$ crossing of the valence bands. (e) Momentum distribution curves at $-250 \mathrm{meV}$ recorded with different incident photon energies from 45 to $95 \mathrm{eV}$. (f) Fermi surface intensity plot measured on the (010) surface at $24 \mathrm{~K}$ showing a small hole pocket at $\Gamma$. The directions of $k_{x}$ and $k_{y}$ are parallel and perpendicular to the $\mathrm{ZrTe}_{3}$ chains in the (010) plane, respectively, while $k_{z}$ is along the (010) surface normal direction.

conventional method to observe the band structure slightly above $E_{F}$ is to broaden the Fermi-Dirac (FD) distribution function by raising the temperature. Surprisingly, the chemical potential steadily shifts upwards with raising the sample temperature. Figure 2(b) exhibits that the chemical potential lies above the top of the valence bands at $200 \mathrm{~K}$. This up-shifting of the chemical potential with increasing temperature is possibly caused by the highly asymmetrical DOS across $E_{F}$, since a similar effect has been observed in Fe-based superconductors [31-33]. An alternative explanation for the energy shift with temperature is the bandbending effect, as observed in Bi-based TI materials [34,35].

As seen in Fig. 2(b), signals from the conduction bands are still unobserved in the pristine $\mathrm{ZrTe}_{5}$ crystals even at high temperature. We thus perform ARPES measurements on the $\mathrm{ZrTe}_{5}$ crystals with $0.25 \%$ (nominal value) of $\mathrm{Zr}$ substituted by Ta. The substitution is expected to dope electrons and thus shift the chemical potential upwards. The ARPES results of the substituted $\mathrm{ZrTe}_{5}$ crystals recorded at $190 \mathrm{~K}$ are summarized in Fig. 3. To illustrate the effects of the substitution on the band structure, we plot the extracted bands along $\Gamma-X$ at $200 \mathrm{~K}$ of the pristine $\mathrm{ZrTe}_{5}$ on top of the data of the substituted $\mathrm{ZrTe}_{5}$ in 
Fig. 3(a). Note that the extracted bands of the pristine $\mathrm{ZrTe}_{5}$ are shifted down by $50 \mathrm{meV}$ to match the band dispersions of the substituted $\mathrm{ZrTe}_{5}$. The substitution does not lead to other noticeable changes in the band dispersions, except that it shifts the chemical potential upwards by $50 \mathrm{meV}$. The band topology is thus not changed by this light substitution. From the intensity plot along $\Gamma-X$ shown in Fig. 3(a), we can distinguish some weak signals above the holelike band top. After division by the FD distribution function convoluted with the energy resolution Gaussian function set in the ARPES measurements, the signals above $E_{F}$ are much enhanced, as shown in Figs. 3(c) and 3(d). We identify one peak at $60 \mathrm{meV}$ above $E_{F}$ at the $\Gamma$ point and very weak signals at $k_{x} \sim \pm 0.4 \AA^{-1}$ along $\Gamma-X$. Moreover, the ARPES data we measure along $Y-M$ exhibit weak signals above $E_{F}$ at $k_{x}=0.15 \AA^{-1}$. These signals correspond to the bottoms of the conduction bands, which are in agreement with the bulk band calculations [24]. The ARPES results clearly indicate that the lowest bottom of the conduction bands is located at $\Gamma$, leading to a direct band gap of $\sim 100 \mathrm{meV}$, which is consistent with the STS results (a)

(c)

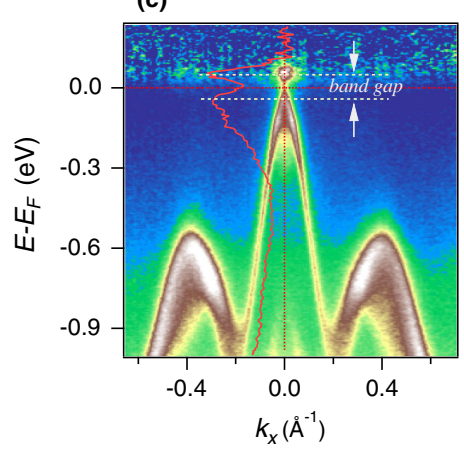

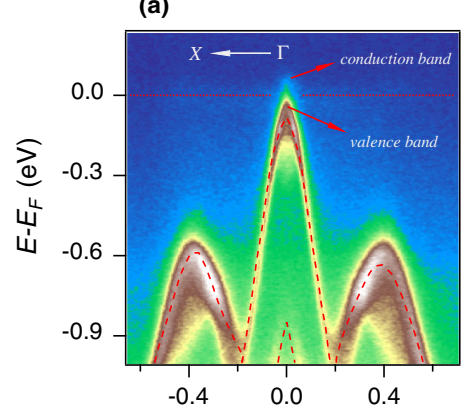

(b)

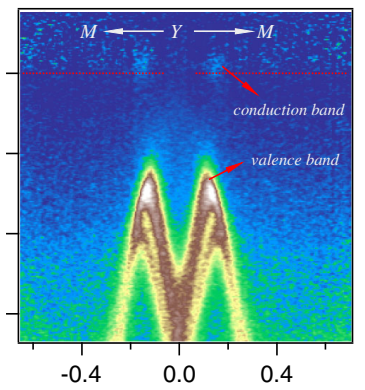

(d)

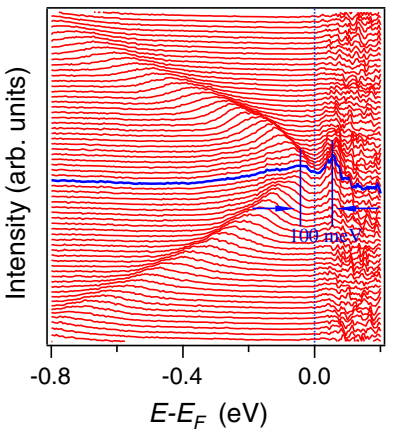

FIG. 3. Band structure of lightly substituted $\mathrm{ZrTe}_{5}$ with a nominal composition $\left(\mathrm{Zr}_{0.9975} \mathrm{Ta}_{0.0025}\right) \mathrm{Te}_{5}$ recorded at $190 \mathrm{~K}$. (a) Intensity plot of the band dispersions along $\Gamma-X$. Dashed curves represent the extracted bands of the pristine $\mathrm{ZrTe}_{5}$ recorded at $200 \mathrm{~K}$, which are shifted down by $50 \mathrm{meV}$ to match the band dispersions of the substituted one. (b),(c) Intensity plots of the band dispersions along $Y-M$ and $\Gamma-X$, respectively, divided by the FD distribution function $(T=190 \mathrm{~K})$ convoluted with the energy resolution Gaussian function $(\Delta E=25 \mathrm{meV})$. The red curve in (c) is the energy distribution curve at $\Gamma$. (d) Energy distribution curve plot of (c). shown in the inset of Fig. 1(c). While the high-resolution STS spectrum exhibits zero DOS within the energy gap at low temperature, the weak but finite spectral weight within the gap in the ARPES data in Fig. 3(c) can be attributed to the shortening of the quasiparticle lifetime at high temperatures, which appears as broadening of the energy distribution peaks in Fig. 3(d).

According to the previous theoretical prediction [24], the monolayer $\mathrm{ZrTe}_{5}$ is a 2D TI, its 3D stacked form is either a weak or strong TI, depending on the strength of the interlayer coupling [36]. Both our STS and ARPES spectra indicate that no surface state exists within the gap on the (010) surface and thus rules out the possibility of a 3D strong TI. As discussed above, if the $\mathrm{ZrTe}_{5}$ crystal is a 3D weak TI stacked by 2D TI sheets, topologically nontrivial helical edge states can emerge inside the gap at the step edge of a top surface layer.

Because of the weak interchain coupling in the $a-c$ plane, the cleavage occurs not only between the adjacent $\mathrm{ZrTe}_{5}$ layers but also between the $\mathrm{ZrTe}_{3}$ and zigzag Te-Te chains, leaving the step edges parallel to the chain direction along the $a$ axis. To check whether the edge states exist, we perform STS measurements along a line crossing the step edge perpendicularly. The inset of Fig. 4(a) shows the measured step topography and the locations of the data points registered with an interval of $1 \mathrm{~nm}$. The step-height difference is measured to be $7.2 \AA$, which is consistent with the $\mathrm{x}$-ray diffraction data for the spacing between the two adjacent $\mathrm{ZrTe}_{5}$ layers, indicating that the upper terrace is a monolayer. In Fig. 4(a), both of the spectra away from the step edge on the lower (blue curves) and upper (green curves) terraces show an indubitable full energy gap of $\sim 100 \mathrm{meV}$. In contrast, the spectra around the step edge (red curves) have a finite conductance within the gap. In the vicinity of the step edge, the gap edges are shifted to lower energy by $\sim 50 \mathrm{meV}$ continuously from the upper terraces to the lower one, possibly due to the slight band-bending effect, whereas the gap size remains unchanged. To see the evolution of the in-gap states near the step edge more clearly, we zoom in on the spectra taken from the 12th to 22 nd points in an energy range covering the energy gap and offset them with a constant incremental conductance in Fig. 4(c). It can be clearly seen that the conductance increases upon approaching the edge from both sides and reaches a maximum right at the edge (point no. 17).

To illuminate the nature of the observed edge states, we calculate the electronic states of freestanding ribbon having left (terminated with Te-Te zigzag chain) and right (terminated with $\mathrm{ZrTe}_{3}$ chain) edges and compare the results with that of the ribbon supported by a thick $\mathrm{ZrTe}_{5}$ slab, which mimics the situation for step edges. We find that the obtained edge states from the freestanding ribbon are qualitatively the same as those from the supported ribbon due to the weak interlayer coupling, as explained in more detail in the Appendix. The results for the freestanding ribbon are 

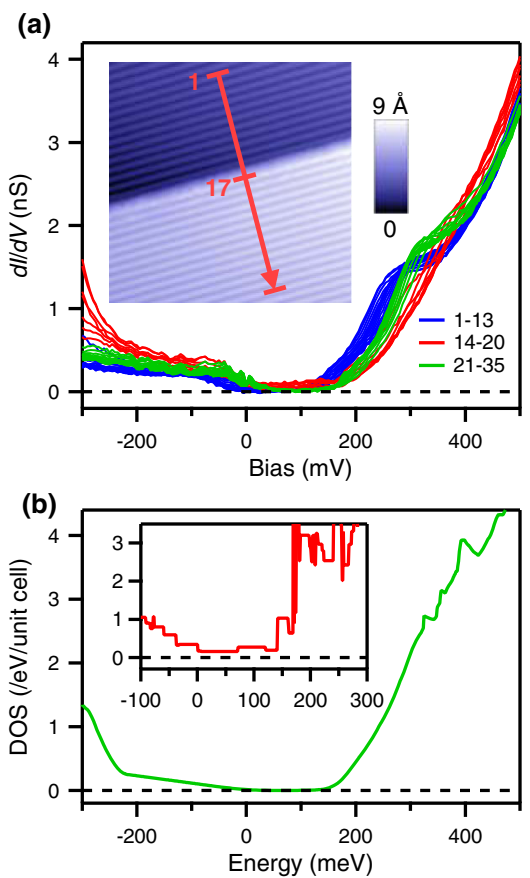

(c)

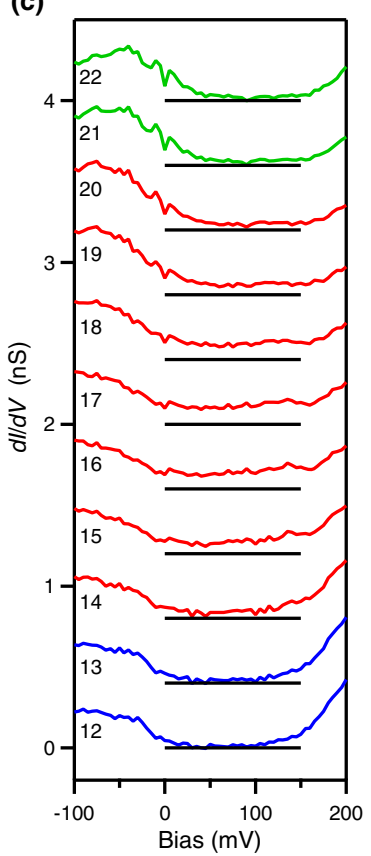

(d)
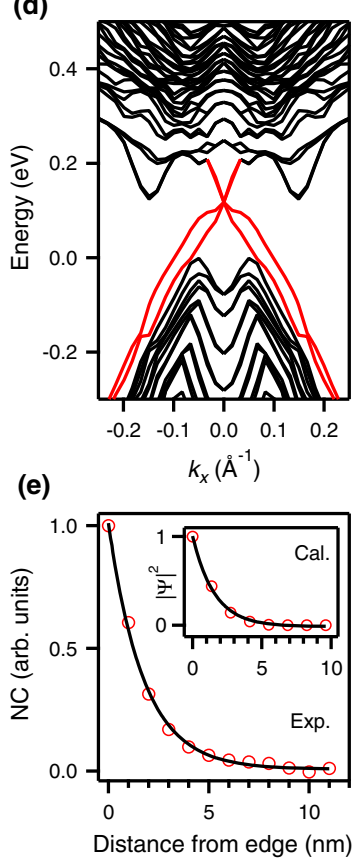

FIG. 4. (a) Tunneling differential conductance spectra along a line perpendicular to a monolayer step edge $(I=500 \mathrm{pA}$, $V=500 \mathrm{mV}$ ). Red curves are tunneling spectra measured near the step edge, while the blue and green curves are measured away from the step edge on the lower and upper terraces, respectively. The inset shows the topographic image of the step $(I=30 \mathrm{pA}$, $V=300 \mathrm{mV}$ ) and data point locations. (b) Calculated DOS of the top monolayer. The inset shows the calculated DOS of the edge states of the monolayer along the chain direction. (c) Evolution of the edge states. For clarity, the spectra from the 12th to 22nd points are offset with equal intervals of $0.4 \mathrm{nS}$. Black lines indicate the zero-conductance level for each spectrum. (d) Calculated band dispersions of the edge states of the monolayer along the chain direction. (e) Normalized conductance integral within the gap plotted as a function of the distance from the edge. The black curve is an exponential function fit to the data with a characteristic decay length $\xi=1.5 \mathrm{~nm}$. The inset plots the calculated mode of the wave function for the edge state close to $\Gamma$ as a function of the distance from the edge. The black curve is an exponential function with a characteristic decay length $\xi=1.6 \mathrm{~nm}$.

presented in Fig. 4(d), in which the Dirac-like edge states in the gap confirm its topologically nontrivial nature. The splitting in the lower branch is due to the nonequivalent left and right edges. As seen in the inset of Fig. 4(b), such Diraclike edge states have a nearly constant DOS inside the gap region, which reproduces the experimentally observed edge states very well. Figure 4(b) also shows the calculated local DOS from the top layer on a (010) slab with a thickness of nine layers, which largely mimics the STS measurements far from the step edge, such as for points no. 1 and no. 35. It exhibits a large energy gap of $\sim 70 \mathrm{meV}$ and reproduces the experimental observations well.

To specify the spatial distribution of the edge states, the normalized conductance integrals within the energy gap for individual spectra are plotted as a function of the distance from the edge and fitted with an exponential function, as shown in Fig. 4(e). The exponential decay of the edge states, with a characteristic length $\xi=1.5 \mathrm{~nm}$, indicates that the observed edge states distribute closely along the step edge and extend laterally merely on the scale of a primitive cell. Such an exponential decay of the edge states is also well reproduced by our calculations with a characteristic length $\xi=1.6 \mathrm{~nm}$, as seen in the inset of Fig. 4(e).

\section{CONCLUSION}

Our experimental results clearly demonstrate that the top surface of $\mathrm{ZrTe}_{5}$ is fully gapped with a gap size of $\sim 100 \mathrm{meV}$. Conducting states with a nearly constant DOS inside the gap are detected near the step edge of one monolayer height. The edge states decay exponentially into the bulk with a characteristic length $\xi=1.5 \mathrm{~nm}$. These unique features are remarkably well reproduced by our first-principles calculations, which explicitly point to the topologically nontrivial nature of the edge states. These results suggest that topological edge states exist at the monolayer step edges on the $\mathrm{ZrTe}_{5}$ surface with a large fully opened band gap. Such a large gap with topological edge states is promising for the QSH device and ideal dissipationless wire operating at high temperatures. This will largely stimulate the applications of TIs and propel topotronics as the next generation of microelectronics and spintronics. Furthermore, $\mathrm{ZrTe}_{5}$ crystals with an inherent insulating bulk gap could be fabricated to have many terraces, which can be viewed as a natural QSH device integrated with multiple edge channels. If an $s$-wave superconducting film covers such step edges, 1D topological superconductivity with Majorana bound states at the 


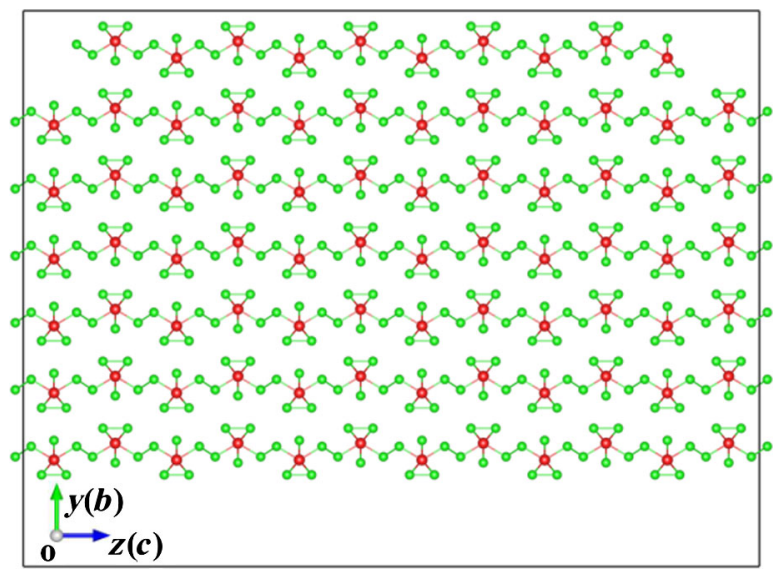

FIG. 5. $\mathrm{A} \mathrm{ZrTe}_{5}$ ribbon terminated with a Te-Te zigzag chain (top left) and a $\mathrm{ZrTe}_{3}$ chain (top right) is put on a six-layer-thick bulk $\mathrm{ZrTe}_{5}$. The width of the ribbon shown here is five c-lattice constants. The interlayer distance is $7.5 \AA$, which leads $3 \mathrm{D} \mathrm{ZrTe}_{5}$ to be in a weak TI [24].
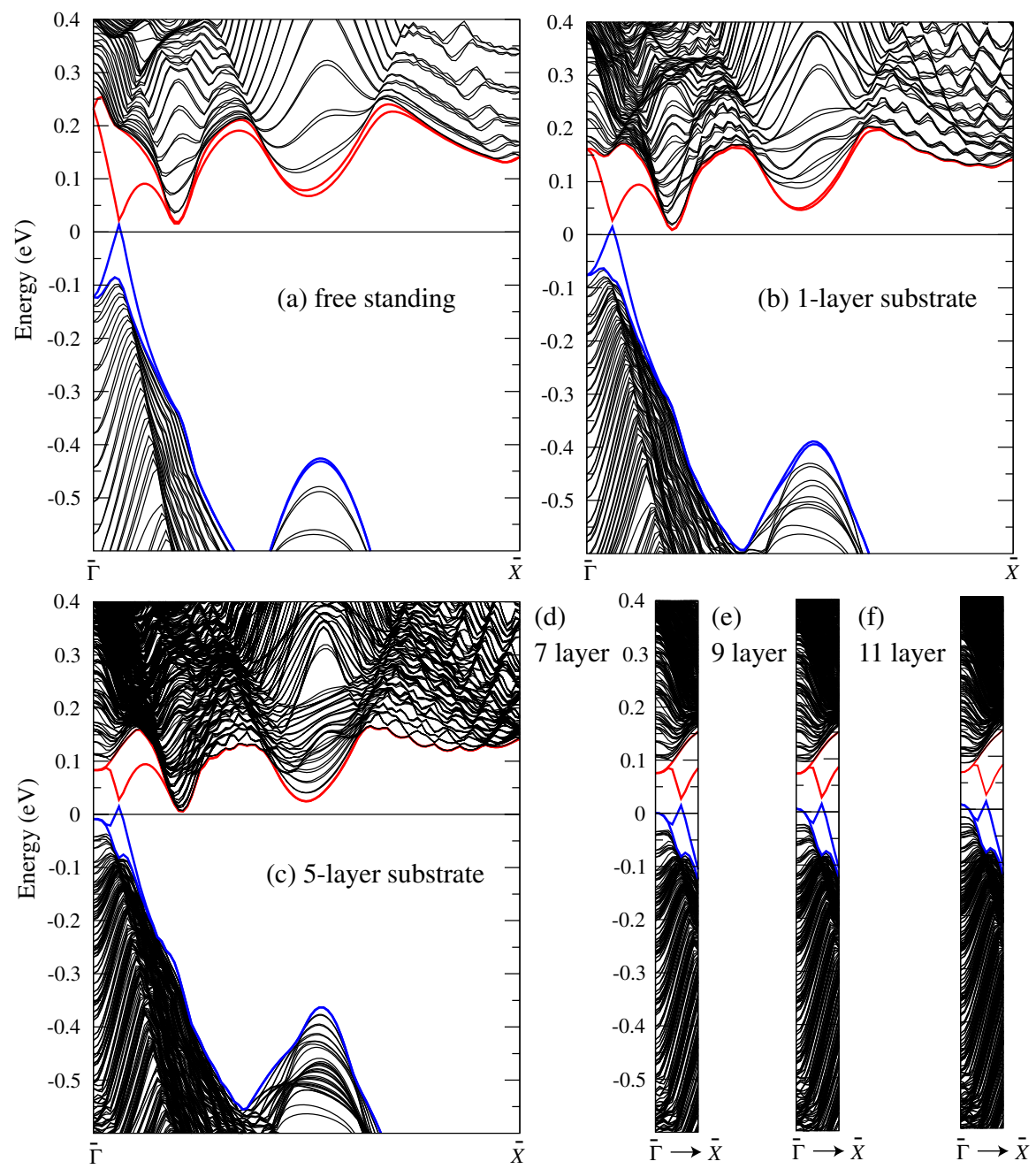

FIG. 6. Tight-binding model band structure along $\Gamma-X$ with fixed ribbon width of $15 c$ unit cells. (a) Freestanding ribbon. (b), (c) Supported with 1- and 5-layer-thick substrates, respectively. (d)-(f) 7-, 9-, and 11-layer-thick substrates, respectively, but only $1 / 10$ of the path from $\Gamma$ to $X$. 


\section{APPENDIX: EDGE STATES OF $\mathrm{ZrTe}_{5}$ RIBBON ON SLAB}

Here, we present the theoretical calculations of a $\mathrm{ZrTe}_{5}$ ribbon, which is either freestanding or supported by 3D bulk $\mathrm{ZrTe}_{5}$. Through this analysis, we show that the edge states of freestanding $\mathrm{ZrTe}_{5}$ ribbon can be well preserved even if it is put on a clean surface of $\mathrm{ZrTe}_{5}$, which simulates the monolayer terrace observed in this paper.

In Fig. 5, the ribbon has its width along the $c$ lattice and runs periodically along the $a$ lattice. It can be obtained from $3 \mathrm{D} \mathrm{ZrTe}_{5}$ once there is a terrace of monolayer height. Such a five- $c$-unit-cell-wide ribbon with six-layer-thick substrate contains 492 atoms. By using first-principles calculations, we check that six layers of $\mathrm{ZrTe}_{5}$ are thick enough to reproduce the 3D bulk band gap. However, to reduce the coupling of the left and right edges of the ribbon to be ignorable, we find that the width larger than nine $c$ unit cells is needed for the freestanding case. For such a wide ribbon with substrate, the total number of atoms is more than 800. It is too demanding for computational effort in first-principles calculations. Therefore, we take the atomic orbital-like Wannier function constructed from the bulk $\mathrm{ZrTe}_{5}$ as the local basis set [24], and a tight-binding Hamiltonian is constructed to simulate the calculation.

The results are presented in Fig. 6. The ribbon width is fixed to be $15 c$ unit cells. The freestanding ribbon has the left- and right-edge states with Dirac-like band structure at $\Gamma$. As the thickness of the substrate increases from 1 to 11 , we find that the Dirac-like edge states are well preserved inside of the band gap. This band gap at $\Gamma$ converges to around $100 \mathrm{meV}$. From these results, we can conclude that the edge states can also exist in the monolayer terrace of $\mathrm{ZrTe}_{5}$.

Finally, in Fig. 4 of the main text, the freestanding ribbon is calculated with cutting-edge potential correction and full electron density self-consistency. Therefore, both the shape and the location of the Dirac-like bands are different from those in Fig. 6, but we think that the main physics of the influence of substrate on the edge states is reproduced here.

[1] C. L. Kane and E. J. Mele, $Z_{2}$ Topological Order and the Quantum Spin Hall Effect, Phys. Rev. Lett. 95, 146802 (2005).

[2] B. A. Bernevig and S.-C. Zhang, Quantum Spin Hall Effect, Phys. Rev. Lett. 96, 106802 (2006).

[3] B. A. Bernevig, T. L. Hughes, and S.-C. Zhang, Quantum Spin Hall Effect and Topological Phase Transition in HgTe Quantum Wells, Science 314, 1757 (2006).

[4] C. Liu, T. L. Hughes, X.-L. Qi, K. Wang, and S.-C. Zhang, Quantum Spin Hall Effect in Inverted Type-II Semiconductors, Phys. Rev. Lett. 100, 236601 (2008).

[5] H. Weng, A. Ranjbar, Y. Liang, Z. Song, M. Khazaei, S. Yunoki, M. Arai, Y. Kawazoe, Z. Fang, and X. Dai, Large-Gap Two-Dimensional Topological Insulator in
Oxygen Functionalized MXene, Phys. Rev. B 92, 075436 (2015).

[6] C.-C. Liu, W. Feng, and Y. Yao, Quantum Spin Hall Effect in Silicene and Two-Dimensional Germanium, Phys. Rev. Lett. 107, 076802 (2011).

[7] Y. Xu, B. Yan, H.-J. Zhang, J. Wang, G. Xu, P. Tang, W. Duan, and S.-C. Zhang, Large-Gap Quantum Spin Hall Insulators in Tin Films, Phys. Rev. Lett. 111, 136804 (2013).

[8] C. Si, J. Liu, Y. Xu, J. Wu, B.-L. Gu, and W. Duan, Functionalized Germanene as a Prototype of Large-Gap Two-Dimensional Topological Insulators, Phys. Rev. B 89, 115429 (2014).

[9] Z. Song, C.-C. Liu, J. Yang, J. Han, M. Ye, B. Fu, Y. Yang, Q. Niu, J. Lu, and Y. Yao, Quantum Spin Hall Insulators and Quantum Valley Hall Insulators of $\mathrm{Bi} X / \mathrm{Sb} X(X=\mathrm{H}, \mathrm{F}, \mathrm{Cl}$ and $\mathrm{Br})$ Monolayers with a Record Bulk Band Gap, NPG Asia Mater. 6, e147 (2014).

[10] W. Luo and H. Xiang, Room Temperature Quantum Spin Hall Insulators with a Buckled Square Lattice, Nano Lett. 15, 3230 (2015).

[11] J.-J. Zhou, W. Feng, C.-C. Liu, S. Guan, and Y. Yao, LargeGap Quantum Spin Hall Insulator in Single Layer Bismuth Monobromide $\mathrm{Bi}_{4} \mathrm{Br}_{4}$, Nano Lett. 14, 4767 (2014).

[12] X. Qian, J. Liu, L. Fu, and J. Li, Quantum Spin Hall Effect in Two-Dimensional Transition Metal Dichalcogenides, Science 346, 1344 (2014).

[13] S. M. Nie, Z. Song, H. Weng, and Z. Fang, Quantum Spin Hall Effect in Two-Dimensional Transition-Metal Dichalcogenide Haeckelites, Phys. Rev. B 91, 235434 (2015).

[14] Z. Song, S. M. Nie, H. Weng, and Z. Fang, Quantum Spin Hall State on Square-like Lattice, arXiv:1508.05220.

[15] Y. Sun, C. Felser, and B. Yan, Graphene-like Dirac States and Quantum Spin Hall Insulators in the Square-Octagonal $M X_{2}(M=\mathrm{Mo}, \mathrm{W} ; X=\mathrm{S}, \mathrm{Se}, \mathrm{Te})$ Isomers, Phys. Rev. B 92, 165421 (2015).

[16] Q. Xu, Z. Song, S. Nie, H. Weng, Z. Fang, and X. Dai, Two-Dimensional Oxide Topological Insulator with IronPnictide Superconductor LiFeAs Structure, Phys. Rev. B 92, 205310 (2015).

[17] Y. Ma, L. Kou, Y. Dai, and T. Heine, Quantum Spin Hall Effect and Topological Phase Transition in TwoDimensional Square Transition Metal Dichalcogenides, Phys. Rev. B 92, 085427 (2015).

[18] M. König, S. Wiedmann, C. Brüne, A. Roth, H. Buhmann, L. W. Molenkamp, X. L. Qi, and S.C. Zhang, Quantum Spin Hall Insulator State in HgTe Quantum Wells, Science 318, 766 (2007).

[19] I. Knez, R. R. Du, and G. Sullivan, Evidence for Helical Edge Modes in Inverted InAs 1/4 Gasb Quantum Wells, Phys. Rev. Lett. 107, 136603 (2011).

[20] F. Yang, L. Miao, Z. F. Wang, M.-Yu Yao, F. Zhu, Y. R. Song, M.-X. Wang, J.-P. Xu, A. V. Fedorov, Z. Sun, G. B. Zhang, C. Liu, F. Liu, D. Qian, C. L. Gao, and J.-F. Jia, Spatial and Energy Distribution of Topological Edge States in Single Bi (111) Bilayer, Phys. Rev. Lett. 109, 016801 (2012).

[21] S. H. Kim, K.-H. Jin, J. Park, J. S. Kim, S.-H. Jhi, T.-H. Kim, and H. W. Yeom, Edge and Interfacial States in a Two-Dimensional Topological Insulator: Bi(111) Bilayer on $\mathrm{Bi}_{2} \mathrm{Te}_{2} \mathrm{Se}$, Phys. Rev. B 89, 155436 (2014). 
[22] I. K. Drozdov, A. Alexandradinata, S. Jeon, S. NadjPerge, H. Ji, R. J. Cava, B. A. Bernevig, and A. Yazdani, One-Dimensional Topological Edge States of Bismuth Bilayers, Nat. Phys. 10, 664 (2014).

[23] C. Pauly, B. Rasche, K. Koepernik, M. Liebmann, M. Pratzer, M. Richter, J. Kellner, M. Eschbach, B. Kaufmann, L. Plucinski, C. M. Schneider, M. Ruck, J. van den Brink, and M. Morgenstern, Subnanometre-Wide Electron Channels Protected by Topology, Nat. Phys. 11, 338 (2015).

[24] H. Weng, X. Dai, and Z. Fang, Transition-Metal Pentatelluride $\mathrm{ZrTe}_{5}$ and $\mathrm{HfTe}_{5}$ : A Paradigm for Large-Gap Quantum Spin Hall Insulators, Phys. Rev. X 4, 011002 (2014).

[25] G. Kresse and J. Furthmüller, Efficiency of Ab-Initio Total Energy Calculations for Metals and Semiconductors Using a Plane-Wave Basis Set, Comput. Mater. Sci. 6, 15 (1996).

[26] G. Kresse and J. Furthmüller, Efficient Iterative Schemes for Ab Initio Total-Energy Calculations Using a Plane-Wave Basis Set, Phys. Rev. B 54, 11169 (1996).

[27] J. P. Perdew, K. Burke, and M. Ernzerhof, Generalized Gradient Approximation Made Simple, Phys. Rev. Lett. 77, 3865 (1996).

[28] H. Fjellvåg and A. Kjekshus, Structural Properties of $\mathrm{ZrTe}_{5}$ and $\mathrm{HfTe}_{5}$ as Seen by Powder Diffraction, Solid State Commun. 60, 91 (1986).

[29] H. Weng, X. Dai, and Z. Fang, Exploration and Prediction of Topological Electronic Materials based on FirstPrinciples Calculations, MRS Bull. 39, 849 (2014).
[30] H. Weng, R. Yu, X. Hu, X. Dai, and Z. Fang, Quantum Anomalous Hall Effect and Related Topological Electronic States, Adv. Phys. 64, 227 (2015).

[31] V. Brouet, P.-H. Lin, Y. Texier, J. Bobroff, A. TalebIbrahimi, P. Le Fèvre, F. Bertran, M. Casula, P. Werner, S. Biermann, F. Rullier-Albenque, A. Forget, and D. Colson, Large Temperature Dependence of the Number of Carriers in Co-Doped $\mathrm{BaFe}_{2} \mathrm{As}_{2}$, Phys. Rev. Lett. 110, 167002 (2013).

[32] R. S. Dhaka, S. E. Hahn, E. Razzoli, R. Jiang, M. Shi, B. N. Harmon, A. Thaler, S. L. Bud'ko, P. C. Canfield, and A. Kaminski, Unusual Temperature Dependence of Band Dispersion in $\mathrm{Ba}\left(\mathrm{Fe}_{1-x} \mathrm{Ru}_{x}\right)_{2} \mathrm{As}_{2}$ and Its Consequences for Antiferromagnetic Ordering, Phys. Rev. Lett. 110, 067002 (2013).

[33] P.-H. Lin, Y. Texier, A. Taleb-Ibrahimi, P. Le Fèvre, F. Bertran, E. Giannini, M. Grioni, and V. Brouet, Nature of the Bad Metallic Behavior of $\mathrm{Fe}_{1.06} \mathrm{Te}$ Inferred from Its Evolution in the Magnetic State, Phys. Rev. Lett. 111, 217002 (2013).

[34] M. Bianchi, D. Guan, S. Bao, J. Mi, B. B. Iversen, P. D. C. King, and P. Hofmann, Coexistence of the Topological State and a Two-Dimensional Electron Gas on the Surface of $\mathrm{Bi}_{2} \mathrm{Se}_{3}$, Nat. Commun. 1, 128 (2010).

[35] E. Frantzeskakis et al., Dirac States with Knobs on: Interplay of External Parameters and the Surface Electronic Properties of Three-Dimensional Topological Insulators, Phys. Rev. B 91, 205134 (2015).

[36] L. Fu and C. L. Kane, Topological Insulators with Inversion Symmetry, Phys. Rev. B 76, 045302 (2007). 